\title{
Neutrophil Gelatinase-Associated Lipocalin as a Marker for Renal Dysfunction Detection in Critically Ill Patients with Increased Intraabdominal Pressure
}

\author{
Claudiu Puiac ${ }^{1}$, Janos Szederjesi2², Alexandra Lazăr², Codruța Bad², Lucian Pușcașiu ${ }^{1}$ \\ 1 Gynecology and Obstetrics Department, University of Medicine and Pharmacy of Tirgu Mures, Romania \\ ${ }^{2}$ Anesthesiology Department, University of Medicine and Pharmacy of Tirgu Mures, Romania
}

\begin{abstract}
Introduction: Elevated intraabdominal pressure (IAP) it is known to have an impact on renal function trough the pressure transmitted from the abdominal cavity to the vasculature responsible for the renal blood flow. Intraabdominal pressure is found to be frequent in intensive care patients and also to be a predictor of mortality. Intra- abdominal high pressure is an entity that can have serious impact on intensive care admitted patients, studies concluding that if this condition progresses to abdominal compartment syndrome mortality is as high as $80 \%$.

Aim: The aim of this study was to observe if a link between increased intraabdominal pressure and modification in renal function exists (NGAL, creatinine clearance).

Material and Method: The study enrolled 30 critically ill patients admitted in the Intensive Care Unit of SCJU Tîrgu Mures between November 2015 and August 2016. The study enrolled adult, hemodynamically stable patients admitted in intensive critical care - defined by a normal blood pressure maintained without any vasopressor or inotropic support, invasive monitoring using PICCO device and abdominal pressure monitoring.

Results: The patients were divided into two groups based on the intraabdominal pressure values: normal intraabdominal pressure group $=52$ values and increased intraabdominal group $=35$ values. We compared the groups in the light of NGAL values, 24 hours diuresis, GFR and creatinine clearance. The groups are significantly different when compared in the light of NGAL values and GFR values. We obtained a statistically significant correlation between NGAL value and 24 hour diuresis. No other significant correlations were encountered between the studied items.

Conclusions: NGAL values are increased in patients with high intraabdominal pressure which may suggest its utility as a cut off marker for patients with increased intraabdominal pressure. There is a significant decreased GFR in patient with elevated intraabdominal pressure, observation which can help in early detection of renal injury in patients due to high intraabdominal pressure. No correlation was found between creatinine clearance and increased intraabdominal pressure.
\end{abstract}

Keywords: neutrophil gelatinase-associated lipocalin, intensive care, renal dysfunction, abdominal pressure, creatinine clearance, diuresis, glomerular filtration rate

Received: 28 November 2016 / Accepted: 15 January 2017

\section{INTRODUCTION}

Elevated intraabdominal pressure (IAP) is known to have an impact on renal function through the pressure transmitted from the abdominal cavity to the vasculature responsible for the renal blood flow [1].

Intraabdominal hypertension (IAH) is frequently encountered in intensive care patients and it was pro- posed to be responsible for AKI - acute kidney injury in these patients [2].

Intraabdominal pressure is found to be also a predictor of mortality [3]. Intraabdominal high pressure is an entity that can have serious impact on intensive care patients, studies concluding that if this condition progresses to abdominal compartment syndrome mortality is as high as $80 \%$ [4]. 
In 2013 the World Society of the Abdominal Compartment Syndrome modified some of the definitions of the intraabdominal pressure and abdominal compartment syndrome, and defined 4 grades of abdominal pressure [5]:

- Grade I, IAP 12-15 mmHg

- Grade II, IAP 16-20 mmHg

- Grade III, IAP 21-25 mmHg

- Grade IV, IAP > 25 mmHg

High intraabdominal pressure can interfere with the normal function of cardiovascular system, pulmonary system and renal system [6,7].

Because it has such high interferences it is important not only to detect the high intraabdominal pressure but also to understand and evaluate its effects on the other systems of the body. The renal system is one of the affected systems and its proper function is vital for critically ill patients. This is why the need to recognize the possible malfunctions caused by intraabdominal increased pressure.

It is known that increased abdominal pressure can reduce the abdominal perfusion pressure which further affects the end organs and tissues. Renal artery and renal veins are affected in the presence of intraabdominal high pressure, and renal injury appears due to ischemia and congestion due to low renal perfusion [8].

Neutrophil gelatinase-associated lipocalin (NGAL) is produced in the distal nephron and its production is increased when an injury appears at this level. Serum concentrations of NGAL increase before those of creatinine. Creatinine and NGAL could be used in determining kidney problems for both patients without known renal disease and for monitoring known disease [9].

There are several methods to determine the renal injury in the context of intraabdominal pressure increase: ultrasound flow probes, which is invasive, CEUS (contrast enhanced ultrasound), which is a non-invasive blood flow measurement but necessitates a special designed ultrasound machine and medical skills [10]. These are direct measurements, but the renal state can be indirectly assessed by measuring some renal products such as urine, urinary creatinine. From these products, with the help of some special designed formulas, the renal activity can be graded. The glomerular filtration rate is considered by Kidney Disease: Improving Global Outcomes (KDIGO) Acute Kidney Injury Work Group to be the best index of kidney function [11].

\section{Objective}

The aim of this study was to assess any correlation between NGAL and increased intraabdominal pressure.

A secondary objective of the study was to evaluate the correlations existing between GFR, creatinine clearance and intraabdominal pressure.

\section{GATERIAL AND METHOD}

The study enrolled 30 critically ill patients admitted in the Intensive Care Unit of SCJU TîrguMures between November 2015 and August 2016. The Ethics Committee of the Hospital approved the conduction of this study and patients or their legal representatives were asked for their written approval to enter the study.

The inclusion criteria were: adult aged patients admitted in intensive critical care, hemodynamically stable - defined by a normal blood pressure maintained without any vasopressor or inotropic support, invasive monitoring using PICCO device and abdominal pressure monitoring.

Exclusion criteria: chronic renal failure.

Study protocol: after patient admission in the intensive care unit, the hemodynamic stability was assessed, the anthropometric parameters were registered, the intraabdominal pressure was measured and renal function was assessed by obtaining the values of serum creatinine, N-GAL. The 24-hour diuresis was recorded. These values were registered for each day the patient stayed in the intensive care unit and were noted in a specially designed sheet.

For the variables measurement we used the following:

- The intraabdominal pressure was measured with the AbViser (ConvaTec, Salt Lake City, USA) device - the measurement was done with the help of a urethra-vesical catheter. A value of $>12 \mathrm{mmHg}$ was considered high intraabdominal pressure.

- The NGAL value was measured using the Alere Triage NGAL tests with the Alere Triage MeterPro device ( Alere Inc. USA). Normal values of serum NGAL are between $40-100 \mathrm{ng} / \mathrm{ml}$.

- The creatinine serum value was determined by blood sampling and was processed using routine laboratory tests

- For glomerular filtration ratio calculation we used the Cockroft Gault formula

- GFR for males $=(140$ - age $) \times$ weight $(\mathrm{kg}) / 72 \mathrm{x}$ serum creatinine

- GFR for females= GFR for males $\times 0.85$ 
The study enrolled 30 patients from which we obtained data sheets with the listed parameters. No patients were excluded from the study.

The patients were divided in two groups, based on the value of intraabdominal pressure. The first group included the patients with normal value of the intraabdominal pressure - NP, 52 values, and the second group included the patients with increased value of the intraabdominal pressure - IP, 35 values.

The data were collected using Microsoft Excel (Microsoft, Washington, USA) and statistically processed using the programs GraphPad (GraphPad Software, Inc., California, USA) and MedCalc (MedCalc Software, Ostend, Belgium). We used an alpha significance of 0.05 and statistic tests for non- paired data, parametric and non-parametric tests: Man Whitney, Student's t test for equality of means, and Levene's test for equality of variances. Data series distribution normality was assessed using Kolmogorov-Smirnov goodness-of-fit test.

Table 1. Measured parameters of the enrolled patients

\begin{tabular}{lccc} 
Variable & Minimum & Maximum & Median \\
Age $(\mathrm{yrs})$ & 39 & 84 & 74 \\
NGAL $(\mathrm{ng} / \mathrm{ml})$ & 36 & 1300 & 316 \\
IAP(cmH2O) & 5 & 51 & 10 \\
$24 \mathrm{hr}$ diuresis $(\mathrm{ml})$ & 100 & 7800 & 2700 \\
GFR (ml/hr) & 9 & 103 & 47.5 \\
\hline
\end{tabular}

\section{RESULTS}

We enrolled a total of 30 patients who spent in the ICU a minimum of one day and maximum of 12 days. The measured parameters of the entire group of 30 patients are presented in table 1. Inferential statistics for the two formed groups, showed no statistical significance between them in respect of 24 hour diuresis, creatinine clearance and age.

The groups are significantly different when compared in the light of NGAL values and GFR values. The detailed results are shown in table 2.

We performed Spearman correlation between 24 our diuresis, glomerular filtration rate, NGAL value and intraabdominal pressure. The results are presented in the table 3 .

We obtained a statistically significant correlation between NGAL value and 24-hour diuresis. No other significant correlations were encountered between the studied items.

Table 3. Correlation matrix -Spearman $r$

\begin{tabular}{lcccc} 
& $\begin{array}{c}\mathbf{2 4 h} \\
\text { diuresis }\end{array}$ & $\begin{array}{c}\text { Creatinine } \\
\text { clearance }\end{array}$ & NGAL & GFR \\
\hline 24h & & 0.05 & -0.39 & 0.14 \\
Diuresis & & $(p=0.63)$ & $(p=0.004)$ & $(p=0.15)$ \\
Creatinine & 0.05 & & -0.14 & 0.92 \\
clearance & $(p=0.63)$ & & $(p=0.35)$ & $(p=1.5)$ \\
NGAL & -0.39 & -0.14 & & -0.10 \\
& $(p=0.004)$ & $(p=0.35)$ & & $(p=0.47)$ \\
GFR & 0.14 & 0.92 & -0.10 & \\
\hline & $(p=0.15)$ & $(p=1.5)$ & $(p=0.47)$ & \\
\hline
\end{tabular}

Table 2: Group Statistics for the two studied groups

\begin{tabular}{|c|c|c|c|c|c|c|}
\hline & $\begin{array}{l}\text { Intraabdominal } \\
\text { pressure }\end{array}$ & $\mathbf{N}$ & Mean & $\begin{array}{c}\text { Std. } \\
\text { Deviation }\end{array}$ & $\begin{array}{l}\text { Std. Error } \\
\text { Mean }\end{array}$ & P value /Test used \\
\hline \multirow[t]{2}{*}{ Diuresis } & Normal & 49 & 2709.18 & 1470.03 & 210.005 & $0.65 / T$ test \\
\hline & Increased & 35 & 2861.42 & 1661.06 & 280.77 & \\
\hline \multirow[t]{2}{*}{ Creatinine clearance } & Normal & 44 & 70.61 & 42.48 & 6.40 & \\
\hline & Increased & 33 & 61.34 & 31.18 & 5.42 & 0.27 / T test \\
\hline \multirow[t]{2}{*}{ NGAL } & Normal & 26 & 324.11 & 299.05 & 58.64 & \\
\hline & Increased & 19 & 571.73 & 411.61 & 94.43 & 0.02 / T test \\
\hline \multirow[t]{2}{*}{ GFR } & Normal & 50 & 63.49 & 41.78 & 5.90 & \\
\hline & Increased & 33 & 46.30 & 24.92 & 4.33 & 0.02 / Mann-Whitney \\
\hline \multirow[t]{2}{*}{ Age } & Normal & 52 & 68.23 & 13.99 & 1.94 & \\
\hline & Increased & 35 & 69.31 & 6.69 & 1.13 & $0.6 / T$ test \\
\hline
\end{tabular}




\section{DISCUSSIONS}

Our study shows an inverse significant correlation between NGAL and 24-hour urine output, which can guide the medical approach towards a thorough evaluation of renal function. Also, in this respect we obtained another significant result when we compared the increased intraabdominal pressure and the value of this biomarker.

The effects of increased intraabdominal pressure on the renal system are among the most frequently encountered in the above condition [12].

Neutrophil gelatinase-associated lipocalin (NGAL) is a molecule that is released by polymorphonuclear granulocytes in context of inflammation and its source is the endothelial cells of nephrons for urinary secretion and later reabsorption into bloodstream [13]. A low amount of NGAL circulates in the bloodstream and is freely filtered by the glomerulus. Normal values are between $40-100 \mathrm{ng} / \mathrm{ml}$. [14] Serum NGAL is secreted under stress conditions from epithelial cells, tumor cells, liver, and kidney [15].

Recent developments in the field of biomarkers pointed out the NGAL as an indicator of kidney injury in the context of increased intraabdominal pressure. In correlation with this, several studies suggest that whole blood NGAL is not as sensitive as a marker for renal injury as urinary NGAL $[16,17]$.

Whole blood NGAL may not be superior to RIFLE criteria for predicting mortality for renal injury in critically ill patients [18].

The need for this new rapid determination of kidney impairment in case of increased intraabdominal pressure came to meet the need of more prompt actions of reducing intraabdominal pressure for the secondary lesions to have a lower occurrence, and the patient's prognostic to be improved [19].

The results resemble the ones obtained by other authors, but in experimental studies on mice [20].

We did not find and significant correlations between this biomarker and creatinine clearance or the glomerular filtration rate, but the literature that studied urinary NGAL showed positive correlations between creatinine clearance and glomerular filtration rate [21].

In the subgroup analysis we had two significant differences between the compared groups and those were related to NGAL and GFR. Regarding the NGAL values, in the group with increased pressure we found sig- nificantly higher values of this biomarker than in the one with normal intraabdominal pressure. Also, in the group with increased intraabdominal pressure the GFR is significantly reduced compared to that from the normal intraabdominal pressure.

The literature on this matter is comprised of a multitude of studies which cannot conclude the exact utility for this marker but which can conclude that it could be useful in guiding the therapeutic conduct $[20,22]$. Although the literature is inconclusive about this marker, in critically ill patients with increased intraabdominal pressure it could be a good detector of renal injury, especially because the glomerular filtration rate is also decreased in this group of patients. This points out the pressure effects on the renal blood flow, and early detection of renal injury may improve patient recovery.

\section{- CONCLUSIONS}

NGAL values are increased in patients with high intraabdominal pressure which may suggest its utility in patients with increased intraabdominal pressure.

There is a significantly decreased GFR in patients with elevated intraabdominal pressure, observation that can help in early detection of renal injury in patients due to high intraabdominal pressure.

No correlation was found between creatinine clearance and increased intraabdominal pressure.

\section{- CONFLICT OF INTEREST}

None declared.

\section{DEFERENCES}

1. Bradley SE, Bradley GP. The effect of increased intraabdominal pressure on renal function in man. J Clin Invest. 1947;26:10101022.

2. Mohmand H, Goldfarb S. Renal dysfunction associated with intraabdominal hypertension and the abdominal compartment syndrome. J Am Soc Nephrol. 2011;22:615-621.

3. Malbrain ML, Chiumello D, Pelosi P. Prevalence of intraabdominal hypertension in critically ill patients: a multicentre epidemiological study. Intensive Care Med. 2004;30(5):822-9.

4. Vidal MG, Ruiz Weisser J, Gonzalez F, et al. Incidence and clinical effects of intraabdominal hypertension in critically ill patients. Crit Care Med. 2008;36(6):1823-31.

5. Kirkpatrick AW, Roberts DJ, De Waele J, et al. Intraabdominal hypertension and the abdominal compartment syndrome: 
28 - The Journal of Critical Care Medicine 2017;3(1)

updated consensus definitions and clinical practice guidelines from the World Society of the Abdominal Compartment Syndrome. Intensive Care Medicine. 2013;399(7):1190-1206.

6. Puiac C, Szederjesi J, Lazar A, Rad P, Almasy E, Pușcasiu L. Influence of Ventilation Parameters on Intraabdominal Pressure. The Journal of Critical Care Medicine 2016;2(2):8084

7. Sui F, Zheng Y, Li WX. Renal circulation and microcirculation during intraabdominal hypertension in a porcine model. Eur Rev Med Pharmacol Sci. 2016;20(3):452-61

8. Balogh ZJ, Lumsdaine W, Moore EE. Postinjury abdominal compartment syndrome: from recognition to prevention. Lancet. 2014;384(9952):1466-1475

9. Prasad D. Neutrophil gelatinase-associated lipocalin Neutrophil gelatinase-associated lipocalin (NGAL). A new marker of kidney disease. Scand J Clin Lab Invest Suppl. 2008;241:89-94

10. Garabed E, Lameire N. KDIGO Clinical Practice Guideline for Acute Kidney Injury. Kidney inter., Suppl. 2012;2:1-138

11. Soni SS, Bobek DC. NGAL: a biomarker of acute kidney injury and other systemic conditions. International Urology and Nephrology. 2010;42(1):141-150

12. Laet IE, Malbrain $M$. Current insights in intraabdominal hypertension and abdominal compartment syndrome. Med Intensiva. 2007;31(2):88-99

13. Devarajan P. Neutrophil gelatinase-associated lipocalin (NGAL): a new marker of kidney disease. Scand J Clin Lab Invest Suppl. 2008;241():89-94

14. Axelsson L, Bergenfeldt M, Ohlsson K. Studies of the release and turnover of a human neutrophil lipocalin. Scand J Clin Lab Invest. 1995; 55(7):577-88
Available online at: www.jccm.ro

15. Cai L, Rubin J, Han $W$ et al. The origin of multiple molecular forms in urine of HNL/NGAL. Clin J Am Soc Nephrol. 2010; 5(12):2229-35.

16. Cai L, Borowiec J, Xu S, Han W, Venge P. Assays of urine levels of $\mathrm{HNL} / \mathrm{NGAL}$ in patients undergoing cardiac surgery and the impact of antibody configuration on their clinical performances. Clin Chim Acta. 2009; 403:121.

17. Glassford NJ, Schneider AG, Xu S, et al. The nature and discriminatory value of urinary neutrophil gelatinaseassociated lipocalin in critically ill patients at risk of acute kidney injury. Intensive Care Med. 2013;39:1714.

18. Linko $R$, Pettila $V$, Kuitunen $A$, et al. Plasma neutrophil gelatinase-associated lipocalin and adverse outcome in critically ill patients with ventilatory support. Acta Anaesthesiol Scand. 2013;57:855.

19. L. Ke, H.B. Ni, Z.H. Tong, et al. The importance of timing of decompression in severe acute pancreatitis combined with abdominal compartment syndrome. J Trauma Acute Care Surg. 2013;74:1060-1066

20. Hjortrup $P$ B, Haase $N$, Wetterslev $M$ et al. Clinical review: Predictive value of neutrophil gelatinase-associated lipocalin for acute kidney injury in intensive care patients. Crit Care. 2013;17(2):211.013

21. Patel ML, Sachan R, Verma A, Kamal R, Gupta KK. Neutrophil gelatinase-associated lipocalin as a biomarker of disease progression in patients with chronic kidney disease. Indian J Nephrol. 2016;26(2):125-30.

22. Munna L P,RekhaS,Misra M, RitulK, RadheyS,Pushpalata S. Prognostic significance of urinary NGAL in chronic kidney disease. Int J NephrolRenovasc Dis. 2015;8:139-144. 